\title{
On fault propagation in deterioration of multi-component systems
}

\author{
Zhenglin Liang*, Ajith Kumar Parlikad, Rengarajan Srinivasan, Nipat Rasmekomen \\ ${ }^{*}$ Correspondence to: Institute for Manufacturing, 17 Charles Babbage Road, Cambridge CB3 0FS, United Kingdom. E-mail address: zl284@ cam.ac.uk
}

\begin{abstract}
In extant literature, deterioration dependence among components can be modelled as inherent dependence and induced dependence. We find that the two types of dependence may co-exist and interact with each other in one multi-component system. We refer to this phenomenon as fault propagation. In practice, a fault induced by the malfunction of a non-critical component may further propagate through the dependence amongst critical components. Such fault propagation scenario happens in industrial assets or systems (bridge deck, and heat exchanging system). In this paper, a multi-layered vector-valued continuous-time Markov chain is developed to capture the characteristics of fault propagation. To obtain the mathematical tractability, we derive a partitioning rule to aggregate states with the same characteristics while keeping the overall aging behaviour of the multi-component system. Although the detailed information of components is masked by aggregated states, lumpability is attainable with the partitioning rule. It means that the aggregated process is stochastically equivalent to the original one and retains the Markov property. We apply this model on a heat exchanging system in oil refinery company. The results show that fault propagation has a more significant impact on the system's lifetime comparing with inherent dependence and induced dependence.
\end{abstract}

Keywords: Markov processes, Reliability, Risk analysis, and Stochastic processes

\section{Notation}

$v$ : The number of critical components.

$X_{0}(t)$ : The of vector of the system condition in normal system deterioration process at time.

$X_{l, 0}(t)$ : The deterioration state $l^{\text {th }}$ critical component in normal system deterioration process at time $t$.

$w_{l, 0}(t)$ : Deterioration rate of $l^{\text {th }}$ critical component in normal system deterioration process at time $t$.

$r_{l, 0}$ : The intrinsic deterioration rate of critical component in the normal system deterioration process.

$\lambda_{n i, 0}$ : Normal deterioration rate between the $i^{\text {th }}$ systemic state and the $(i+1)^{t h}$ systemic state.

$X_{h}(t)$ : The of vector of the system condition component in the fault propagation caused by the $h^{\text {th }}$ type of malfunction on non-critical components.

$X_{l, h}(t)$ : The deterioration state $l^{t h}$ critical component in the fault propagation caused by the $h^{\text {th }}$ type of malfunction on non-critical components.

$w_{l, h}(t)$ : Deterioration rate of $l^{t h}$ critical component in the fault propagation caused by the $h^{\text {th }}$ type of malfunction on non-critical components.

$r_{l, h}$ : The intrinsic deterioration rate of critical component in the $h^{\text {th }}$ fault propagation.
$\mathcal{L}$ : Partitioning rule for Markov aggregation.

$\lambda_{d i, h}$ : The deterioration rate of $h^{\text {th }}$ fault propagation between the $i^{\text {th }}$ state and the $(i+1)^{\text {th }}$ state, where $\lambda_{d i, h}>\lambda_{n i, 0}$.

$\lambda_{f i, h}$ : Rate of $h^{\text {th }}$ type of malfunction on non-critical components at systemic state $i$.

$Y_{i, 0}(t): i^{\text {th }}$ aggregated state in normal system deterioration process at time $t$.

$Y_{i, h}(t): i^{t h}$ Aggregated state in the fault propagation caused by the $h^{\text {th }}$ type of malfunction on non-critical components. $f_{f}(t)$ : Failure time distribution of a multi-component system with fault propagation.

$f_{h}(t)$ : Failure time distribution of a multi-component system with inherent dependence.

$\Delta_{f}$ : Lifetime reduction caused by fault propagation.

$\Delta_{d}$ : Lifetime reduction caused by induced dependence.

$\Delta_{h}$ : Lifetime reduction caused by inherent dependence.

\section{Introduction}

Advances made by the reliability engineering community in modelling the deterioration of multi-component systems have significantly improved the ability to manage and maintain the multi-component systems. Such models consider the deterioration processes of individual components as well as the dependence among them.Three 
types of dependencies have been identified: economic dependence, structural dependence, and stochastic dependence [1]. Economic dependence and structural dependence express the opportunities and constraints in managing and maintaining the multi-component system. Stochastic dependence is defined by Dekker [1] as where "... the state of a component influences the lifetime distribution of other components". It is a critical factor that needs to be considered when modelling the deterioration of multi-component systems. In [2], stochastic dependence is further classified into failure dependence and degradation dependence, the last of which is the focus of this paper. In extant literature, two different types of approaches are used to describe the degradation dependence, namely induced dependence and inherent dependence.

(1) Induced dependence indicates damage to other components caused by the malfunction of one component. It highlights the directional causality between malfunctioned component and influenced components. The typical model to express the induced dependence is shock damage model. The literature within this category are [3]-[7].

(2) Inherent dependence is an underlying interactive deterioration mechanism among aging components because their operational and functional interactions, such as load sharing. Unlike the induced dependence, inherent dependence focuses the deterioration dependence in a way of co-degradation. Multivariate distribution and copulas are two prevalent approaches to model the inherent dependence. References for inherent dependence are [8]-[12].

For multi-component systems composed of critical components and non-critical components, in normal situations, the deteriorations of critical components may correlate with each other in the manner of inherent dependence. When non-critical components are damaged by an exogenous event and becomes malfunctioned, it may change the operating environment of critical components so that they are no longer deteriorating under their rated condition. Therefore, a malfunctioned non-critical component could result in the accelerated deterioration of one or more of the critical components and may subsequently damage their interconnected critical components, thus causing the system to deteriorate faster than normal pace. In this paper, this scenario is referred as fault propagation, which represents the interdependence of inherent dependence and induced dependence. It is an interdependence between inherent dependence and induced dependence - a "meta-dependence". Fault propagation with the meta dependence characteristic is not sufficiently explored in the literature. However, some industrial systems are experiencing the fault propagation. A typical example can be found in concrete bridge decks. The concrete bridge deck is a primary element that needs to be considered for bridge maintenance. It may be subject to the attack of chloride ion, which is regarded as one of the most significant factors in progressive deck deterioration. The chloride ion may penetrate through the crack and accelerate the deterioration of the reinforcing bars which matches with the characteristic of induced dependence. Because reinforcing bars share the load, the damage on one steel reinforcing bar will increase the load on others and accelerate their deterioration. This scenario fits in the scenario of inherent dependence. The induced chloride ion can further propagate through inherent dependence and accelerate the deterioration of concrete deck. This indicates the practical existence of fault propagation scenario. Based on the observation of field experts, fault propagation can have a significant influence on the lifetime.

Because of the significant impact of fault propagation in multi-component systems, it starts to gain more attention in the field of reliability engineering. The dominant threat of transient fault propagation in networked control systems is also analysed by an ontology-based fault propagation analysis [13]. The balance between redundancy and working sharing is optimized by genetic algorithm. Huang et al. have proposed a hierarchical scheme for analysing the transient fault propagation in brake-by-wire systems [14]. The scheme can detect and mitigate the transient fault propagation on both node level with a signature based detection and system level with an anomaly based method. Xing and Levitin have developed a combinatorial method for evaluating the exact reliability of binary-state systems subject to competing propagated failure and failure isolation [15]. They have also developed an approach by universal generating function and reliability block diagram for assessing the performance of the series-parallel multi-state system with propagated failures having selective effect [16]. In [17], the approach has been improved by considering the randomness of failure propagation time. Peng et al. have considered both redundancy and working sharing in a series-parallel multistate system with multi-fault coverage [18]. In [19], an integrated safety prognosis model has been developed based on the dynamic Bayesian network to model the complex system with fault propagation. The risk of the fault propagation is estimated by an ant colony algorithm. Yang and Aldemir have proposed a computationally efficient approach based on Markov/cell-to-cell mapping technique for tracing the fault propagation throughout the system [20]. A pruning algorithm is developed to control the complexity reduction rate. Verlinden et al. have designed a hybrid approach by combining static reliability block diagram with continuous-time Markov chain (CTMC) for a dynamic system with redundancy and online repair [21]. The approach can overcome the state space explosion problem caused by CTMC. As a synthesis of the recent literature, most of the studies have demonstrated the dynamic statespace model is a powerful technique for modelling multicomponent systems with fault propagation. However, such type of model may encounter the state space explosion problem. The existing approaches for the state space and complexity reduction normally induces errors to the system.

This paper differentiates itself by exploring the metadependent features of fault propagation in multi-component systems. In addition, we have derived a partitioning rule to attain the ideal, albeit theoretical, lumpability for the 
deterioration model. It can effectively reduce the size of state space without inducing error while keeping the Markov property on the aggregated process. The rest of paper is structured as follow: we model the deterioration of multicomponent systems as a multi-layered vector-valued CTMC in Section 2. Then, a theorem is provided to aggregate the state space of the model and achieve a more compact model for further study. Section 3 discusses and analyses the impact of fault propagation on the lifetime with an illustrative example. The exact lifetime distribution is expressed analytically. The result is verified by an extreme case scenario. Section 4 applies the deterioration model to a heat exchanging system. The impact of fault propagation on the system's lifetime is calculated and compared with the inherent dependence and induced dependence. The sensitivity of the parameters of the fault propagation is analysed. Section 5 summarizes the concluding remarks of this paper.

\section{Modelling fault propagation}

\section{$2.1 \quad$ System description}

We study the multi-component system consisting of critical components and non-critical components. The multicomponent system may subject to fault propagation during their lifetime. The critical component is essential to system's operation and directly related to the system's condition. The condition of critical components is deteriorating over time. The deteriorating rate of the critical component is related to the condition of other critical components and whether the non-critical component is functioning properly or not. The non-critical components are indirectly related to the system's condition. However, if the non-critical component is malfunctioned, it will increase the deterioration rate of critical components. We denote the accumulated deterioration on all critical components with index $i$. The operation of system will be interrupted by maintenance or replacement due to the risk of system failure, if $i$ pass beyond a given threshold $\zeta$.

\subsection{Modeling approach}

We develop an $m$ layered vector-valued CTMC to model the deterioration process of the multi-component system. The layer with $m=0$ indicates the system in normal deterioration process. The layers with $m>0$ indicate the system is in process of fault propagation caused by the different types of malfunctioned non-critical components. The condition of the system is modelled as a vector of critical components' conditions. We formulate the inherent dependence in a way of deterioration rate interactions. For a system with $v$ critical components, $\left\{X_{0}(t)\right\}=$ $\left\{X_{1,0}(t), X_{2,0}(t), \ldots, X_{v, 0}(t)\right\} \quad$ expresses the normal deterioration process. The deterioration of the $l^{\text {th }}$ critical component is represented by the state transition process $\left\{X_{l, 0}(t)\right\}, X_{l, 0}(t)=0$ indicates that the critical component $l$ is in the "new" state. When the damage and deterioration accumulate, $X_{l, 0}(t)$ increases. Note that the deterioration of critical components consists of their independent natural deterioration as well as any influences from the condition of other critical components. The deterioration rate $\omega_{l, 0}(t)$ is shown as Eq. (1).

$$
\omega_{l, 0}(t)=r_{l, 0}+\sum_{j \in\{1,2, \ldots, v\} / l} g\left(X_{j, 0}(t)\right)
$$

$\omega_{l, 0}(t)$ is constructed by two additive parts, namely the intrinsic deterioration rate $r_{l, 0}$ and affected deterioration rate, which is a linear function $g(x)$ of other critical components' conditions $X_{j, 0}(t)$. Comparing with [22], we have refined the expression of inherent dependence by extending the affected deterioration rate to a linear function of other critical components' conditions.

The deterioration rate of critical component might also be influenced by different malfunctioned non-critical components. We use $h$ to index the different types of malfunctions on non-critical components. The rate of the $h^{\text {th }}$ type of malfunctions is related to the overall accumulated deterioration amongst all critical components and is signified as $\beta_{i, h}$. After the $h^{\text {th }}$ malfunction, the system will transit to $h^{\text {th }}$ layer to represent the fault propagation. The fault propagation is expressed as $\left\{X_{h}(t)\right\}=\left\{X_{1, h}(t), X_{2, h}(t), \ldots, X_{v, h}(t)\right\}$. Under the influence the $h^{\text {th }}$ type of malfunctions, the deterioration of $l^{\text {th }}$ critical component is denoted $\omega_{l, h}(t)$. We assume that such influence causes intrinsic deterioration rate $r_{l, 0}$ to change to $r_{l, h}$. In general, the deterioration rate of a critical component can be affected by two causes. The first is the deterioration of other critical components. The second is the malfunction of non-critical component.

\subsection{State space aggregation}

Based on the system description, the state space of the $m$ layered vector-valued CTMC increases dramatically with $\zeta$ and $v$ and linearly with $m$.

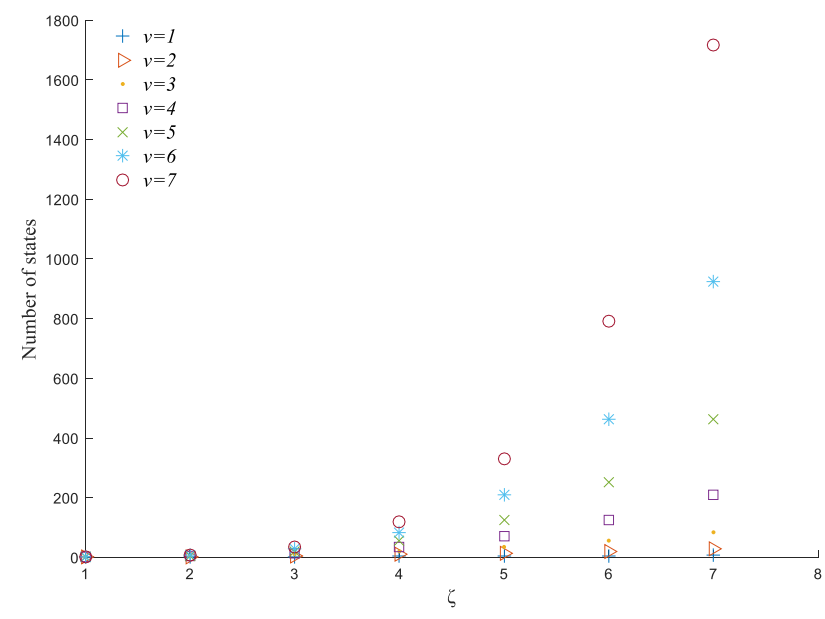

Fig. 1. Nonlinear increase of states with $\zeta$ and $v$

Fig. 1 demonstrates the dramatically nonlinear increase of state space by increasing $\zeta$ and $v$. When $\zeta=7$ and $v=$ 7 , the number of states in one layer is 1716 . The overall number of states for the $m$ layered vector-valued CTMC is 
$1716(m+1)$. This implies that when the number of critical components and their states are large, a state space explosion problem will be encountered, for which it is normally deemed to be time-consuming and mathematically intractable to study and analyse on such large state space [23].

To alleviate the state space explosion problem, a Markov aggregation technique is implemented. The advantages of Markov aggregation are:

1. It reduces the size of state space by aggregating the original state space into a more compact state space.

2. Under certain partitioning rule, lumpability is attainable. Lumpability is an ideal property that indicates the aggregated state transition process is stochastically equivalent to the original one and the aggregated process retains the Markov property.

In [22], the partitioning rule is derived to aggregate vectorvalued CTMC without considering transitions between different layers of vector-valued CTMC. Hence, the induced dependence is not considered in the previous partitioning rule. To derive a partitioning rule for deterioration model of multi-component system with fault propagation, we refine the partitioning rule to aggregate the $m$ layers vectorvector CTMC while attaining lumpability and retaining the Markov property.

Theorem 1: $\mathcal{X}=\left\{X_{0}(t), X_{1}(t), \ldots, X_{m}(t)\right\}$ is a $m$ layers of vector-valued CTMC. Each $\left\{X_{h}(t)\right\}$ contains $v$ elements $\quad\left\{X_{h}(t)\right\}=\left\{X_{1, h}(t), X_{2, h}(t), \ldots, X_{v, h}(t)\right\}$. $\left\{X_{l, h}(t): 1 \leq l \leq v\right\}$ evolves monotonically with a rate

$$
\omega_{l, h}(t)=\lim _{\Delta t \rightarrow 0} \frac{\mathbb{P}\left(X_{l, h}(t+\Delta t)=a+1 \mid X_{l, h}(t)=a, a \in \mathbb{Z}^{*}\right)}{=r_{l, h}+\sum_{j \in\{1,2, \ldots, v\} / l}^{\Delta t} g\left(X_{j, h}(t)\right)}
$$

where $X_{l, h}(0)=0$ and $g(x)$ is a linear function of $X_{j, h}(t)$. We denote $i=\sum_{l=1}^{v} X_{l, h}(t)$, the evolves terminates when $i$ reaches to a predefined threshold $\zeta$.Transition rate between different layers of vector-valued CTMC is directional and only available from $\left\{X_{0}(t)\right\}$ to other layers. The transition rate follows the equation

$$
\begin{aligned}
& \lim _{\Delta t \rightarrow 0} \frac{\mathbb{P}\left(X_{h}(t+\Delta t) \mid X_{0}(t), \sum_{l=1}^{v} X_{l, 0}(t)=i\right)}{\Delta t} \\
& =\left\{\begin{aligned}
\beta_{i, h}, & X_{h}(t+\Delta t)=X_{0}(t) h \neq 0 \quad i<\zeta \\
0, & \text { otherwise }
\end{aligned}\right.
\end{aligned}
$$

Therefore, $\mathcal{X}$ is lumpable with respect to a set of partitioning rules $\mathcal{L}=\left\{\ell_{0}, \ell_{1}, \ldots, \ell_{m}\right\}$ while $\ell_{h}$ expresses as

$$
\ell_{h}=\left\{Y_{i, h}(t)=\left\{X_{h}(t) \mid \sum_{l=1}^{v} X_{l, h}(t)=i\right\}, 0 \leq i \leq \zeta\right\}
$$

$\left\{Y_{i, h}(t)\right\}$ is the lumped serial process of $\left\{X_{h}(t)\right\}$ and the transition rate between $Y_{i, h}$ and $Y_{i+1, h}$ is

$$
\lambda_{i, h}(t)=\sum_{l=1}^{v} r_{l, h}+g(i(v-1))
$$

The transition rate between $Y_{i, 0}$ and $Y_{i, h}$ is identical to $\beta_{i, h}$. Consequently, $\mathcal{Y}=\left\{Y_{i, h}(t), \forall 0 \leq i \leq \zeta, 0 \leq h \leq\right.$ $m\}$ is CTMC with multiple dependent paths. (The proof is shown in appendix).

The aggregation masks the conditions of critical components. It can sufficiently alleviate the state space explosion problem. After the aggregation, the number of states becomes irrelevant with $v$ and linearly increase with $\zeta$ and $m$. The number of states when $\zeta=7$ and $v=7$ is $7(m+1)$. It is a significant reduction on state space comparing with the $1716(m+1)$ states in the original $m$ layered vector-valued CTMC. For a better understanding of the model, we will demonstrate the overall approach on an illustrative example.

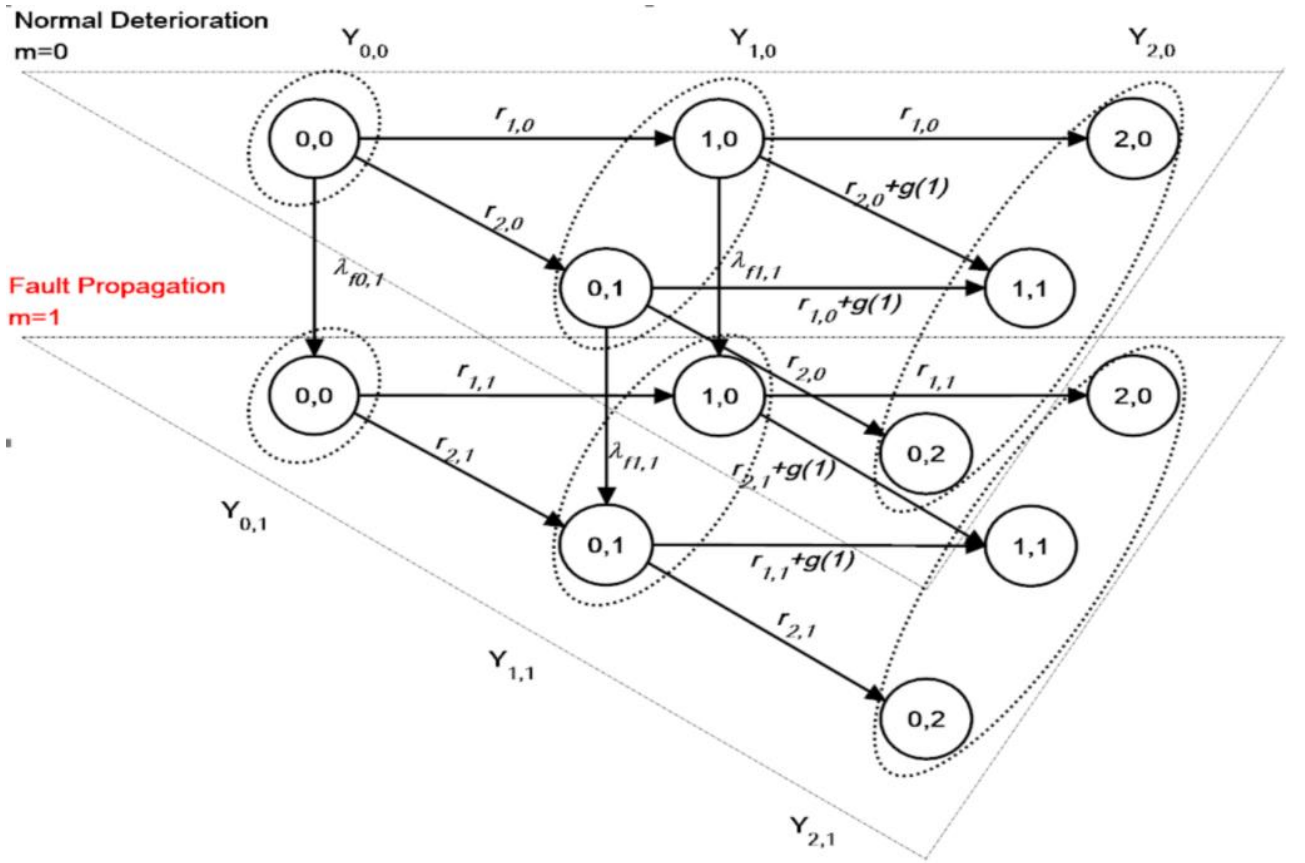

Fig.2. State transition diagram of fault propagation model using two layers of vector-valued CTMC 


\section{Analysis and discussion}

\subsection{Illustrative example}

For the convenience of illustration, a simple example is provided to demonstrate the abovementioned logic of fault propagation in multi-component systems. It considers a multi-component system with two critical components and may subject to one fault propagation scenario caused by the malfunction of a non-critical component. If the accumulated deterioration amongst the critical components reaches to $\zeta=2$, the system is stopped from functioning. The state transition diagram of the multi-component system is illustrated in Figure 2.

In Fig. 2, the horizontal transition is governed by the principle of inherent dependence and vertical transition represents the induced dependence. The model contains 12 states. By applying the Theorem 1, a more compact model for the system is achieved. This aggregated model is referred to as a multi-dependent deteriorating paths model. Figure 3 shows the aggregated state transition diagram of the two layers of vector-valued CTMC in Fig. 2.

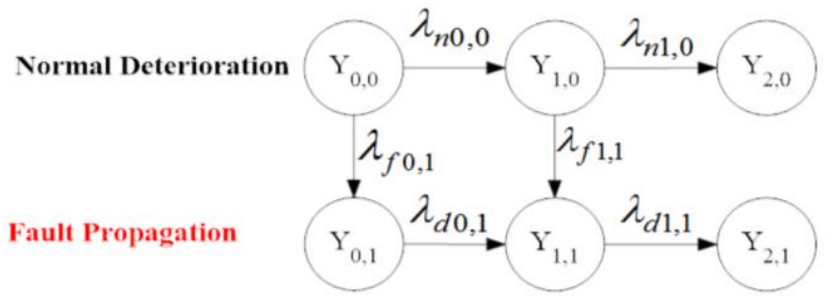

Fig. 3. Multi-dependent deteriorating paths model

Comparing with Fig. 2, the size of state space is halved in the multi-dependent deteriorating paths model. In this way, the component-level model can be successfully aggregated to a more compact system-level model. The parameters in the multi-dependent deteriorating paths model can be calculated by the theorem 1 as shown in Table 1 .

Table 1 Conversion of parameters aggregated model

\begin{tabular}{cc}
\hline $\begin{array}{c}\text { System level } \\
\text { parameters }\end{array}$ & $\begin{array}{c}\text { Component level } \\
\text { Expression }\end{array}$ \\
\hline$\lambda_{n 0,0}$ & $\sum_{l=1}^{2} r_{l, 0}$ \\
$\lambda_{n 1,0}$ & $\sum_{l=1}^{2} r_{l, 0}+g(1)$ \\
$\lambda_{d 0,1}$ & $\sum_{l=1}^{2} r_{l, 1}$ \\
$\lambda_{d 1,1}$ & $\sum_{l=1}^{2} r_{l, 1}+g(1)$ \\
\hline \hline
\end{tabular}

Through this aggregation process, the $m$ layers vector valued CTMC model has been transformed into a multidependent deteriorating path model. It helps the model to regain the mathematical tractability and opens up a successful way to express and analyse the impact of fault propagation on the lifetime of multi-component systems.

3.2 Calculate and analyse the impact of fault propagation We study the impact of fault propagation on the lifetime of multi-component systems using a phase-type distribution.
To examine the impact of fault propagation in an articulated manner, we implement the aggregated multi-dependent deteriorating paths model. Without loss of generality, the absorbing state $Y_{2,0}$ and $Y_{2,1}$ could be combined into one state. From Table 1, it is clear that for modelling the metadependence of fault propagation, the deterioration rates of multi-component system should be heterogeneous. Therefore, it is more generalized and representative than [7] by considering the heterogeneity of deterioration rates caused by inherent dependence amongst the critical components. The probabilistic behavior of the absorbing CTMC can be summarized and characterized by the infinitesimal generator [24]. The infinitesimal generator is a $5 \times 5$ matrix $\boldsymbol{Q}$.

$$
\boldsymbol{Q}=\left[\begin{array}{ccccc}
-\left(\lambda_{n 0,0}+\lambda_{f 0,1}\right) & \lambda_{f 0,1} & \lambda_{n 0,0} & 0 & 0 \\
0 & -\lambda_{d 0,1} & 0 & \lambda_{d 0,1} & 0 \\
0 & 0 & -\left(\lambda_{n 1,0}+\lambda_{f 1,1}\right) & \lambda_{f 1,1} & \lambda_{n 1,0} \\
0 & 0 & 0 & -\lambda_{d 1,1} & \lambda_{d 1,1} \\
0 & 0 & 0 & 0 & 0
\end{array}\right]
$$

The model contains four transient states and one absorbing state. Transient rate is the rate to transfer from a transient state to another transient state. Absorbing rate is the rate transfer from a transient state to absorbing state. Therefore, the infinitesimal generator matrix can also be represented by a combination of submatrices $\mathbf{S}$ and $\mathbf{S}^{0}$.

$$
\boldsymbol{Q}=\left[\begin{array}{cc}
\boldsymbol{S} & \boldsymbol{S}^{\mathbf{0}} \\
\mathbf{0} & 0
\end{array}\right]
$$

$\boldsymbol{S}$ contains the transition rate between transient states and $\mathbf{S}^{0}$ represents the transition intensities from transient rate to absorbing rate.

$$
\begin{gathered}
\mathbf{S}=\left[\begin{array}{cccc}
-\left(\lambda_{n 0,0}+\lambda_{f 0,1}\right) & \lambda_{f 0,1} & \lambda_{n 0,0} & 0 \\
0 & -\lambda_{d 0,1} & 0 & \lambda_{d 0,1} \\
0 & 0 & -\left(\lambda_{n 1,0}+\lambda_{f 1,1}\right) & \lambda_{f 1,1} \\
0 & 0 & 0 & -\lambda_{d 1,1}
\end{array}\right] \\
\mathbf{S}^{0}=\left[\begin{array}{c}
0 \\
0 \\
\lambda_{n 1,0} \\
\lambda_{d 1,1}
\end{array}\right]
\end{gathered}
$$

The lifetime distribution of the multi-component system can be calculated using a phase-type distribution as Buchholz [25] has derived:

$$
f_{f}(t)=\boldsymbol{\varphi} e^{\mathbf{S} t} \mathbf{S}^{0}
$$

Where $f(t)$ indicates the lifetime function of a multicomponent system with fault propagation, and $\boldsymbol{\varphi}$ is the probability vector denoting the initial probability of starting in any of the four states. The value of $\boldsymbol{\varphi}$ can be assigned based on the inspected condition of the system. Then, the Eq. (2) can be used to estimate the remaining life time of the system. As the focus of this section is on analysing the impact of fault propagation on the lifetime of multicomponent system, we assume the system starts at the as good as new state, therefore, $\boldsymbol{\varphi}$ is $\left[\begin{array}{llll}1 & 0 & 0 & 0\end{array}\right]$. As $\mathbf{S}$ is an upper triangular matrix, it has four roots: $\lambda_{1}=-\left(\lambda_{n 0,0}+\right.$ $\left.\lambda_{f 0,1}\right), \quad \lambda_{2}=-\lambda_{d 0,1}, \quad \lambda_{3}=-\left(\lambda_{n 1,0}+\lambda_{f 1,1}\right)$ and $\lambda_{4}=$ $-\lambda_{d 1,1}$. The eigenvectors matrix $\mathbf{S}$ can be calculated as. 


$$
\begin{aligned}
& \left(\mathbf{S}-\lambda_{1} \mathbf{I}\right) \mathbf{V}_{1}=0 \\
& \mathbf{V}_{1}=\left[\begin{array}{llll}
1 & 0 & 0 & 0
\end{array}\right]^{T} \\
& \left(\mathbf{S}-\lambda_{2} \mathbf{I}\right) \mathbf{V}_{2}=0 \\
& \mathbf{V}_{2}=\left[\begin{array}{llll}
1 & \frac{\lambda_{n 0,0}+\lambda_{f 0,1}-\lambda_{d 0,1}}{\lambda_{f 0,1}} & 0 & 0
\end{array}\right]^{T} \\
& \left(\mathbf{S}-\lambda_{3} \mathbf{I}\right) \mathbf{V}_{3}=0 \\
& \mathbf{V}_{3}=\left[\begin{array}{llll}
1 & 0 & \frac{\lambda_{n 0,0}+\lambda_{f 0,1}-\left(\lambda_{n 1,0}+\lambda_{f 1,1}\right)}{\lambda_{n 0,0}} & 0
\end{array}\right]^{T} \\
& \left(\mathbf{S}-\lambda_{4} \mathbf{I}\right) \mathbf{V}_{4}=0 \\
& \mathbf{V}_{4}=\left[\begin{array}{c}
1 \\
\frac{\lambda_{d 0,1}\left(\lambda_{n 1,0}+\lambda_{f 1,1}-\lambda_{d 1,1}\right)\left(\lambda_{n 0,0}+\lambda_{f 0,1}-\lambda_{d 1,1}\right)}{\lambda_{f 0,1} \lambda_{d 0,1}\left(\lambda_{n 1,0}+\lambda_{f 1,1}-\lambda_{d 1,1}\right)+\lambda_{n 0,0} \lambda_{f 1,1}\left(\lambda_{d 0,1}-\lambda_{d 1,1}\right)} \\
\frac{\lambda_{f 1,1}\left(\lambda_{d 0,1}-\lambda_{d 1,1}\right)\left(\lambda_{n 0,0}+\lambda_{f 0,1}-\lambda_{d 1,1}\right)}{\lambda_{f 0,1} \lambda_{d 0,1}\left(\lambda_{n 1,0}+\lambda_{f 1,1}-\lambda_{d 1,1}\right)+\lambda_{n 0,0} \lambda_{f 1,1}\left(\lambda_{d 0,1}-\lambda_{d 1,1}\right)} \\
\frac{\left(\lambda_{d 0,1}-\lambda_{d 1,1}\right)\left(\lambda_{n 1,0}+\lambda_{f 1,1}-\lambda_{d 1,1}\right)\left(\lambda_{n 0,0}+\lambda_{f 0,1}-\lambda_{d 1,1}\right)}{\lambda_{f 0,1} \lambda_{d 0,1}\left(\lambda_{n 1,0}+\lambda_{f 1,1}-\lambda_{d 1,1}\right)+\lambda_{n 0,0} \lambda_{f 1,1}\left(\lambda_{d 0,1}-\lambda_{d 1,1}\right)}
\end{array}\right] \\
& f_{f}(t)=\left[\frac{-\lambda_{n 0,0} \lambda_{n 1,0}}{\lambda_{n 0,0}+\lambda_{f 0,1}-\lambda_{n 1,0}-\lambda_{f 1,1}}+\frac{\lambda_{f 0,1} \lambda_{d 0,1} \lambda_{d 1,1}}{\lambda_{d 0,1}-\lambda_{d 1,1}}\left(\frac{1}{\lambda_{n 0,0}+\lambda_{f 0,1}-\lambda_{d 0,1}}-\frac{1}{\lambda_{n 0,0}+\lambda_{f 0,1}-\lambda_{d 1,1}}\right)\right. \\
& \left.+\frac{\lambda_{n 0,0} \lambda_{f 1,1} \lambda_{d 1,1}}{\lambda_{n 1,0}+\lambda_{f 1,1}-\lambda_{d 1,1}}\left(\frac{1}{\lambda_{n 0,0}+\lambda_{f 0,1}-\lambda_{n 1,0}-\lambda_{f 1,1}}+\frac{1}{\lambda_{n 0,0}+\lambda_{f 0,1}-\lambda_{d 1,1}}\right)\right] e^{-\left(\lambda_{n 0,0}+\lambda_{f 0,1}\right) t} \\
& -\frac{\lambda_{f 0,1} \lambda_{d 0,1} \lambda_{d 1,1}}{\left(\lambda_{n 0,0}+\lambda_{f 0,1}-\lambda_{d 0,1}\right)\left(\lambda_{d 0,1}-\lambda_{d 1,1}\right)} e^{-\lambda_{d 0,1} t}+\frac{\lambda_{n 0,0}}{\lambda_{n 0,0}+\lambda_{f 0,1}-\lambda_{n 1,0}-\lambda_{f 1,1}}\left(\lambda_{n 1,0}\right. \\
& \left.-\frac{\lambda_{f 1,1} \lambda_{d 1,1}}{\lambda_{n 1,0}+\lambda_{f 1,1}-\lambda_{d 1,1}}\right) e^{-\left(\lambda_{n 1,0}+\lambda_{f 1,1}\right) t} \\
& +\left[\frac{\lambda_{d 1,1}}{\lambda_{n 0,0}+\lambda_{f 0,1}-\lambda_{d 1,1}}\left(\frac{\lambda_{f 0,1} \lambda_{d 0,1}}{\lambda_{d 0,1}-\lambda_{d 1,1}}+\frac{\lambda_{n 0,0} \lambda_{f 1,1}}{\lambda_{n 1,0}+\lambda_{f 1,1}-\lambda_{d 1,1}}\right)\right] e^{-\lambda_{d 1,1} t} \\
& \mathbf{J}=\left[\begin{array}{cccc}
-\left(\lambda_{n 0,0}+\lambda_{f 0,1}\right) & 0 & 0 & 0 \\
0 & -\lambda_{d 0,1} & 0 & 0 \\
0 & 0 & -\left(\lambda_{n 1,0}+\lambda_{f 1,1}\right) & 0 \\
0 & 0 & 0 & -\lambda_{d 1,1}
\end{array}\right] \\
& e^{\mathrm{J} t}=\left[\begin{array}{cccc}
e^{-\left(\lambda_{n 0,0}+\lambda_{f 0,1}\right) t} & 0 & 0 & 0 \\
0 & e^{-\lambda_{d 0,1} t} & 0 & 0 \\
0 & 0 & e^{-\left(\lambda_{n 1,0}+\lambda_{f 1,1}\right) t} & 0 \\
0 & 0 & 0 & e^{-\lambda_{d 1,1} t}
\end{array}\right]
\end{aligned}
$$

Eq. (4) indicates the failure time distribution of such type of systems is a weighted hyper-exponential distribution composed by two different type of exponential terms: one is the sum of the normal deterioration rate and the induced deterioration rate, the other is the accelerated deterioration rate. From the system lifetime perspective, it implies that the induced dependence rate is as important as the normal distribution rate.

The benefit of recognising the impact of fault propagation is twofold. In the long term, it can reduce the risk of system failures caused by the gross overestimation of the system's lifetime. In the short term, it can improve the accuracy of predicting the optimal time for maintenance, by better understanding the potential risk caused by fault propagation.

\subsection{Verification}

According to mathematical modelling research methodology [26], some mathematical models could reduce to previously designed model under an extreme case scenario. This can verify and validate the model. In this subsection, the lifetime equation $f_{f}$ is verified by extreme scenarios. Based on the schematic diagrams in Fig. 3 , when rates of $\lambda_{f 1,0}$ and $\lambda_{f 1,1}$ are approaching to 0 , the schematic diagram degenerates to a single deteriorating path model with heterogeneous deterioration rate between states as illustrated in Fig. 4.

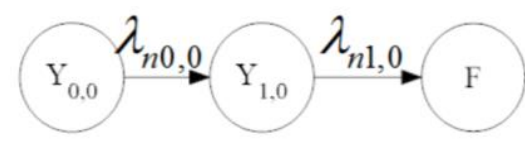

Fig. 4. Deterioration model with inherent dependence In this case, the density of the failure time distribution has been proved to be a hypo-exponential distribution [25], which is expressed in general form as:

$$
f_{h}(t)=\frac{\lambda_{n 0,0} \lambda_{n 1,0}}{\lambda_{n 0,0}-\lambda_{n 1,0}}\left(e^{-\lambda_{n 1,0} t}-e^{-\lambda_{n 0,0} t}\right)
$$

The analytical result of $f_{h}(t)$ is identical to the result when $\lim _{\lambda_{f 1,0} \rightarrow 0, \lambda_{f 1,1} \rightarrow 0} f_{f}(t)$. Hence, Eq. (4) can be partially verified. This simplified scenario has been implemented in the existing articles [27] and [28] to describe the deterioration of a single-unit system. 


\section{$4 \quad$ Practical case study}

To illustrate the differences between the four failure time distributions with fault propagation, with only induced dependence, with inherent dependence and with no dependence. An example of a heat exchanging system is provided.

In oil refinery process and distillation tower, a heat exchanging system is used to preheat the gas to reaction temperature with the recycled heat. Refinery plants could benefit from this process by saving energy. The system contains three components, which are two gas tubes $(v=$ 2) and one desalter. Gas tubes are critical components of the system. High-temperature gas can transfer through the tubes before reaching the cold box. During this process, the heat could be recycled and reused. As the gas pass through, the unwanted materials and particles in the feed gases could deposit and accumulate on the inner surface of the tubes and cause the fouling problem. The fouling could reduce the gases throughput and thermal conductivity which may result in low system operation efficiency. We classify the fouling process into three states, which are new, deposition consolidation and clogged $\left(\left\{X_{0}(t)\right\}=\{0,1,2\}\right)$. When either one of tubes is clogged, or both of tubes are in the deposition consolidation states, we assume the heat exchanging system reaches to the critical point for cleaning. The two tubes are subject to inherent dependence in a manner deterioration state rate interaction as [12] explored and elaborated. The fouling process can be measured by the pressure the gas tubes. The pressure is directly related to the cross-area of the gas tube and in turn related to the fouling process. Desalter is used to remove contaminants from the gas by desalting and dehydration. It is a non-critical component, which has a function of mitigating the polymerisation fouling of the tubes. However, desalter might be malfunctioned because loss chemical injection rate or sludge build up. The fault propagation scenario is defined as when the desalter is malfunctioned, it will accelerate the polymerization fouling of the tubes and catalyse the fouling between the two tubes through the underlying inherent dependence $(m=1)$. Based on the description, the vector conditions of the two gas tubes can be formulated by the $\mathcal{X}$. The state of aggregated process $\mathcal{Y}$ indicates the overall accumulated deposit in the two tubes. The parameter setting for the heat exchanging system is derived from the results in [12] with additional experience values on malfunction rate of desalter.

Table 2: Parameters setting for the illustrative example

\begin{tabular}{cc}
\hline \hline Parameter & Value (/days) \\
\hline$r_{0,1}$ & 0.0123 \\
$r_{0,2}$ & 00117 \\
$g(1)$ & 0.0069 \\
$r_{1,1}$ & 0.0245 \\
$r_{1,2}$ & 0.0233 \\
\hline
\end{tabular}

\begin{tabular}{ll}
\hline \hline$\lambda_{f 0,1}$ & 0.005 \\
$\lambda_{f 1,1}$ & 0.005 \\
\hline \hline
\end{tabular}

The failure time distribution of the system with fault propagation can be calculated by the Eq. (4). To make a comparison, we also calculated the failure time distributions under induced dependence, inherent dependence, and independence scenarios. We can calculate the failure time distribution with induced dependence with the finding in [7]. The failure time distribution with inherent dependence can be calculated by Eq. (5). The failure time distribution under the independent assumption is an Erlang distribution. The four failure time distributions are plotted in Fig. 5 .

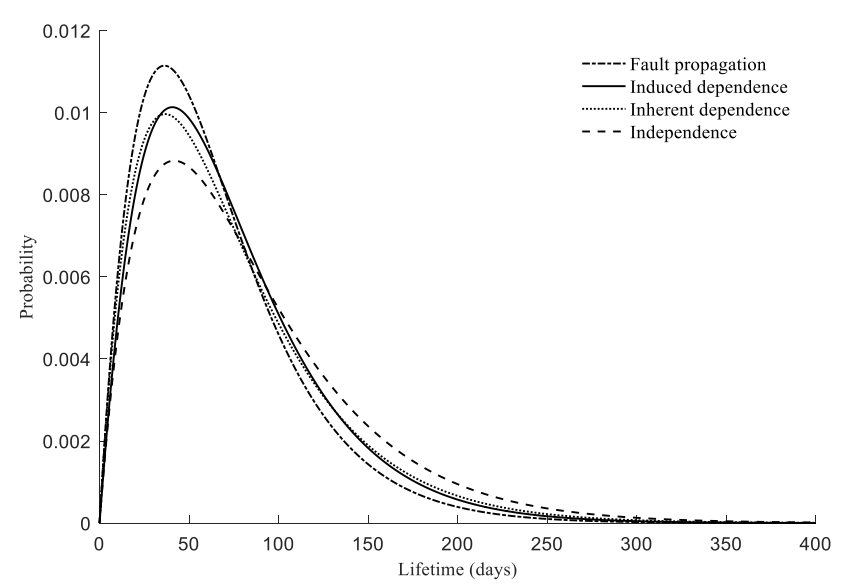

Fig.5. Difference between four failure time distributions

It is clear that the failure time distribution when considering fault propagation has more risk on deterioration failure on early stage than the rest of the three failure time distributions. For comparison, the expected lifetime $T$ under different assumptions can be calculated by Eq. (6)

$$
\mathbb{E}(T)=\int_{0}^{\infty} t f(t) d t
$$

The resulting expected lifetimes under different assumptions are listed in Table 3.

Table 3: Expected lifetime under different assumptions

\begin{tabular}{cc}
\hline \hline Parameter & Result (days) \\
\hline $\mathbb{E}\left(T_{f}\right)$ & 66.367 \\
$\mathbb{E}\left(T_{d}\right)$ & 73.177 \\
$\mathbb{E}\left(T_{h}\right)$ & 74.029 \\
$\mathbb{E}\left(T_{I}\right)$ & 83.333 \\
\hline \hline
\end{tabular}

We use $\mathbb{E}\left(T_{I}\right)$ as the benchmark. Therefore, the lifetime reduction caused by fault propagation $\Delta_{f}$, induced dependence $\Delta_{d}$ and inherent dependence $\Delta_{h}$ are expressed as below:

$$
\begin{aligned}
& \Delta_{f}=\left|\mathbb{E}\left(T_{f}\right)-\mathbb{E}\left(T_{I}\right)\right|=16.966 \text { days } \\
& \Delta_{d}=\left|\mathbb{E}\left(T_{d}\right)-\mathbb{E}\left(T_{I}\right)\right|=10.304 \text { days } \\
& \Delta_{h}=\left|\mathbb{E}\left(T_{h}\right)-\mathbb{E}\left(T_{I}\right)\right|=9.3039 \text { days }
\end{aligned}
$$


Therefore, we can compare the impacts of fault propagation, induced dependence and inherent dependence as shown in Eq. (7).

$$
\Delta_{f}>\Delta_{d}, \Delta_{h}
$$

The impact of fault propagation on expected lifetime is more significant than induced dependence and inherent dependence. It indicates a higher risk for a multi-component system subject to fault propagation. Additionally, it is observable that $\Delta_{f}$ is not identical to the sum of $\Delta_{d}$ and $\Delta_{h}$. This justifies explicitly modelling of the metadependence characteristic of fault propagation.

The impact of fault propagation is controlled by the joint effect of the three types of parameters, which are the affected deterioration rate $g(x)$ the malfunction rate $\lambda_{f i, h}$ and the difference between the fault propagation rate $\lambda_{d i, h}$ and normal deterioration rate $\lambda_{n i, 0}$. We now investigate the sensitivity of fault propagation parameters on the expected lifetime of the system. Sensitivity analysis of the fault propagation parameters is numerically demonstrated based on the same illustrative case. During the test, each parameter is gradually increased. The resulting expected lifetime of the system is plotted against the incremental percentage of the individual parameters in Fig. 6.

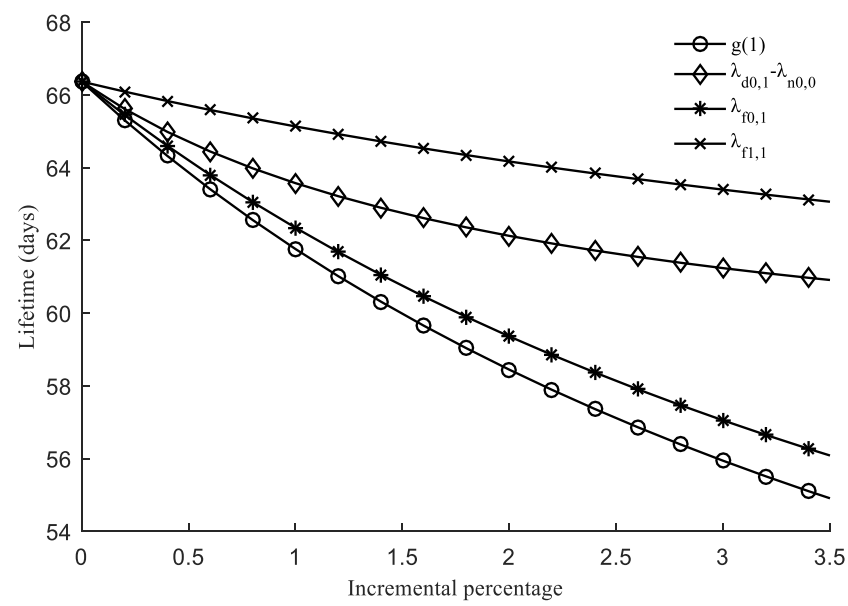

Fig. 6. Sensitivity analysis of fault propagation parameters From Fig. 6, it can be seen that the system's lifetime is more sensitive to the affected deterioration rate and early state malfunction rate. Therefore, to prolong the lifetime of the system, it is worthwhile to aware the fault propagation in the early state of system deterioration.

\section{Conclusion}

To formulate the meta-dependent characteristic of fault propagation, we developed a deterioration model to capture the fault propagation that is a meta-dependence between the inherent dependence and induced dependence. It contributed theoretically to the modelling of stochastic dependence by extending the modelling of dependence to meta-dependence.

The deterioration of multi-component systems is modelled by a $m$ layered vector-valued CTMC. Such type of approach may encounter state space explosion problem. We have derived a partitioning rule to reduce the state space. The number of states in the aggregated process is only linearly proportional to $\zeta$ and $m$. Although the information about the conditions of critical components is masked by the aggregation process, the aggregation by using the partitioning rule induces no error in the overall deterioration model.

The failure time distribution and expected lifetimes are calculated using phase-type distribution. The impact of fault propagation was demonstrated in an industrial case, and its impact was shown to be more significant than induced dependence and inherent dependence. Compared to models under different assumptions, potential benefits of modelling fault propagation were demonstrated.

The current knowledge of fault propagation is mainly in the form of the expert opinion. The designed model is an interface to adopt this knowledge. However, with the increasing attention on the underlying mechanism of the system deterioration, a richer statistical data may be accumulated. It will be beneficial to design a statistical approach.

\section{Appendix}

Proof of Theorem 1:

For a particular $\left\{X_{h}(t)\right\}=\left\{X_{1, h}(t), X_{2, h}(t), \ldots, X_{v, h}(t)\right\}$, because all its elements $X_{l, h}(t)$ evolve in a competing pattern with a rate $\omega_{l, h}(t)$, then according to Finkelstein [29] the rate for any one of the elements as they evolve to its next state is:

$$
\lim _{\Delta t \rightarrow 0} \frac{\mathbb{P}\left(\sum_{l=1}^{v} X_{l, h}(t+\Delta t)=\sum_{l=1}^{v} X_{l, h}(t)+1 \mid X_{h}(t)\right)}{\Delta t}=\sum_{l=1}^{v} \omega_{l, h}(t)
$$

By using this equation to express $\lambda_{i, h}(t)$, we have

$$
\begin{aligned}
\lambda_{i, h}(t) & =\lim _{\Delta t \rightarrow 0} \frac{\mathbb{P}\left(Y_{i+1, h}(t+\Delta t) \mid Y_{i, h}(t)\right)}{\Delta t} \\
& =\lim _{\Delta t \rightarrow 0} \frac{\mathbb{P}\left(\sum_{l=1}^{v} X_{l, h}(t+\Delta t)=i+1 \mid \sum_{l=1}^{v} X_{l, h}(t)=i\right)}{\Delta t} \\
& =\lim _{\Delta t \rightarrow 0} \frac{\mathbb{P}\left(\sum_{l=1}^{v} X_{l, h}(t+\Delta t)=\sum_{l=1}^{v} X_{l, h}(t)+1 \mid \sum_{l=1}^{v} X_{l, h}(t)=i\right)}{\Delta t}
\end{aligned}
$$




$$
\begin{aligned}
& =\frac{\sum_{X_{h}(t) \in Y_{i, h}(t)} \mathbb{P}\left(X_{h}(t) \in Y_{i, h}(t)\right) \lim _{\Delta t \rightarrow 0} \Delta t^{-1} \mathbb{P}\left(\sum_{l=1}^{v} X_{l, h}(t+\Delta t)=\sum_{l=1}^{v} X_{l, h}(t)+1 \mid X_{h}(t)\right)}{\sum_{X_{h}(t) \in Y_{i, h}(t)} \mathbb{P}\left(X_{h}(t) \in Y_{i, h}(t)\right)} \\
& =\frac{\sum_{l=1}^{v} \omega_{l, h}(t) \sum_{X_{h}(t) \in Y_{i, h}(t)} \mathbb{P}\left(X_{h}(t) \in Y_{i, h}(t)\right)}{\sum_{X_{h}(t) \in Y_{i, h}(t)} \mathbb{P}\left(X_{h}(t) \in Y_{i, h}(t)\right)} \\
& =\sum_{l=1}^{v} r_{l, h}+\sum_{j \in\{1,2, \ldots, v\} / l} g\left(X_{j, h}(t)\right) \\
& =\sum_{l=1}^{v} r_{l, h}+g\left(\sum_{j \in\{1,2, \ldots, v\} / l} X_{j, h}(t)\right) \\
& =\sum_{l=1}^{v} r_{l, h}+g(i(v-1)) \\
& \lambda_{f i, h}(t)=\lim _{\Delta t \rightarrow 0} \frac{\mathbb{P}\left(Y_{i, h}(t+\Delta t) \mid Y_{i, 0}(t)\right)}{\Delta t} \\
& =\lim _{\Delta t \rightarrow 0} \frac{\sum_{X_{0}(t) \in Y_{i, 0}(t)} \mathbb{P}\left(X_{0}(t)\right) \mathbb{P}\left(Y_{i, h}(t+\Delta t) \mid X_{0}(t)\right)}{\Delta t \sum_{X_{0}(t) \in Y_{i, 0}(t)} \mathbb{P}\left(X_{0}(t)\right)} \\
& =\lim _{\Delta t \rightarrow 0} \frac{\sum_{X_{0}(t) \in Y_{i, 0}(t)} \mathbb{P}\left(X_{0}(t)\right) \sum_{X_{h}(t+\Delta t) \in Y_{i, h}(t+\Delta t)} \mathbb{P}\left(X_{h}(t+\Delta t) \mid X_{0}(t)\right)}{\Delta t \sum_{X_{0}(t) \in Y_{i, 0}(t)} \mathbb{P}\left(X_{0}(t)\right)} \\
& \sum_{X_{h}(t+\Delta t) \in Y_{i, h}(t+\Delta t)} \mathbb{P}\left(X_{h}(t+\Delta t) \mid X_{0}(t)\right)=\mathbb{P}\left(X_{h}(t+\Delta t) \mid X_{0}(t), X_{h}(t+\Delta t)=X_{0}(t)\right)+\sum_{\substack{X_{h}(t+\Delta t) \in Y_{i, h}(t+\Delta t) \\
X_{h}(t+\Delta t) \neq X_{0}(t)}} \mathbb{P}\left(X_{h}(t+\Delta t) \mid X_{0}(t)\right)=\beta_{i, h} \\
& \lambda_{f i, h}(t)=\frac{\sum_{X_{0}(t) \in Y_{i, 0}(t)} \mathbb{P}\left(X_{0}(t)\right) \lim _{\Delta t \rightarrow 0} \Delta t^{-1} \mathbb{P}\left(X_{h}(t+\Delta t) \mid X_{0}(t), X_{h}(t+\Delta t)=X_{0}(t)\right)}{\sum_{X_{0}(t) \in Y_{i, 0}(t)} \mathbb{P}\left(X_{0}(t)\right)} \\
& =\frac{\sum_{X_{0}(t) \in Y_{i, 0}(t)} \mathbb{P}\left(X_{0}(t)\right) \beta_{i, h}}{\sum_{X_{0}(t) \in Y_{i, 0}(t)} \mathbb{P}\left(X_{0}(t)\right)} \\
& =\beta_{i, h}
\end{aligned}
$$

As we can see, the $\lambda_{i, h}(t)$ only relies on $Y_{i, h}$ and is independent of $X_{l, h}(t) \in X_{h}(t)$ and $\lambda_{f i, h}(t)$ is identical to $\beta_{i, h}$. Hence, $\left\{X_{h}(t)\right\}$ is lumpable with respect to $\ell_{h}$ and the lumped process $\left\{Y_{i, h}(t)\right\}$ is a time homogeneous Markovian process, see [22], [30] and [31].

\section{Reference:}

[1] R. Dekker, R. E. Wildeman, and F. A. van der Duyn Schouten, 'A review of multi-component maintenance models with economic dependence', Math. Methods Oper. Res., vol. 45, no. 3, pp. 411-435, 1997.

[2] N. Rasmekomen and A. K. Parlikad, 'Maintenance Optimization for Asset Systems With Dependent Performance Degradation', IEEE Trans. Reliab., vol. 62, no. 2, pp. 362-367, Jun. 2013.

[3] S.-H. Sheu, T.-H. Liu, Z. G. Zhang, and Y.-H. Chien, 'Extended Optimal Replacement Policy for a TwoUnit System With Shock Damage Interaction', IEEE Trans. Reliab., vol. 64, no. 3, pp. 998-1014, Sep. 2015.

[4] A. Van Horenbeek and L. Pintelon, 'A dynamic predictive maintenance policy for complex multicomponent systems', Reliab. Eng. Syst. Saf., vol. 120, pp. 39-50, 2013.
[5] R. I. Zequeira and C. Bérenguer, 'Periodic imperfect preventive maintenance with two categories of competing failure modes', Reliab. Eng. Syst. Saf., vol. 91, no. 4, pp. 460-468, 2006.

[6] A. Muller, M.-C. Suhner, and B. Iung, 'Formalisation of a new prognosis model for supporting proactive maintenance implementation on industrial system', Reliab. Eng. Syst. Saf., vol. 93, no. 2, pp. 234-253, 2008.

[7] Z. Liang and A. K. Parlikad, 'A Condition-Based Maintenance Model for Assets with Accelerated Deterioration Due to Fault Propagation', IEEE Trans. Reliab., 2015.

[8] H. Peng, D. W. Coit, and Q. Feng, 'Component reliability criticality or importance measures for systems with degrading components', Reliab. IEEE Trans. On, vol. 61, no. 1, pp. 4-12, 2012. 
[9] Y. Wang and H. Pham, 'Modeling the dependent competing risks with multiple degradation processes and random shock using time-varying copulas', Reliab. IEEE Trans. On, vol. 61, no. 1, pp. 13-22, 2012.

[10] L. Bian and N. Gebraeel, 'Stochastic Modeling and Real-Time Prognostics for Multi-Component Systems with Degradation-Rate-Interactions', IIE Trans., no. just-accepted, 2013.

[11] L. Bian and N. Gebraeel, 'Stochastic framework for partially degradation systems with continuous component degradation-rate-interactions', Nav. Res. Logist. NRL, vol. 61, no. 4, pp. 286-303, 2014.

[12] N. Rasmekomen and A. K. Parlikad, 'Condition-based maintenance of multi-component systems with degradation state-rate interactions', Reliab. Eng. Syst. Saf., vol. 148, pp. 1-10, 2016.

[13] C. Zhou, X. Huang, X. Naixue, Y. Qin, and S. Huang, 'A class of general transient faults propagation analysis for networked control systems', IEEE Trans. Syst. Man Cybern. Syst., vol. 45, no. 4, pp. 647-661, Apr. 2015.

[14] S. Huang, C. Zhou, L. Yang, Y. Qin, X. Huang, and $\mathrm{B} . \mathrm{Hu}$, 'Transient fault tolerant control for vehicle brake-by-wire systems', Reliab. Eng. Syst. Saf., vol. 149, pp. 148-163, May 2016.

[15] L. Xing and G. Levitin, 'Combinatorial analysis of systems with competing failures subject to failure isolation and propagation effects', Reliab. Eng. Syst. Saf., vol. 95, no. 11, pp. 1210-1215, 2010.

[16] G. Levitin and L. Xing, 'Reliability and performance of multi-state systems with propagated failures having selective effect', Reliab. Eng. Syst. Saf., vol. 95, no. 6, pp. 655-661, 2010.

[17] G. Levitin, L. Xing, H. Ben-Haim, and Y. Dai, 'Reliability of Series-Parallel Systems With Random Failure Propagation Time', 2013.

[18] R. Peng, H. Mo, M. Xie, and G. Levitin, 'Optimal structure of multi-state systems with multi-fault coverage', Reliab. Eng. Syst. Saf., vol. 119, pp. 18-25, 2013.

[19] J. Hu, L. Zhang, L. Ma, and W. Liang, 'An integrated safety prognosis model for complex system based on dynamic Bayesian network and ant colony algorithm', Expert Syst. Appl., vol. 38, no. 3, pp. 1431-1446, Mar. 2011.

[20] J. Yang and T. Aldemir, 'An algorithm for the computationally efficient deductive implementation of the Markov/Cell-to-Cell-Mapping Technique for risk significant scenario identification', Reliab. Eng. Syst. Saf., vol. 145, pp. 1-8, 2016.

[21] 'Hybrid reliability model for nuclear reactor safety system'. [Online]. Available: http://www.sciencedirect.com/science/article/pii/S09 51832012000063. [Accessed: 13-Dec-2016].
[22] Z. Liang and A. K. Parlikad, 'A tiered modelling approach for condition-based maintenance of industrial assets with load sharing interaction and fault propagation', IMA J. Manag. Math., vol. 26, no. 2, pp. 125-144, 2015.

[23] S. Derisavi, H. Hermanns, and W. H. Sanders, 'Optimal state-space lumping in Markov chains', Inf. Process. Lett., vol. 87, no. 6, pp. 309-315, 2003.

[24] W. J. Stewart, Introduction to the numerical solutions of Markov chains. Princeton Univ. Press, 1994.

[25] P. Buchholz, J. Kriege, and I. Felko, 'Phase-Type Distributions', in Input Modeling with Phase-Type Distributions and Markov Models, Springer, 2014, pp. 5-28.

[26] Z. Huan, 'Mathematical Modelling as a Research Methodology’, Model. Res. Methodol., p. 61, 2010.

[27] S. V. Amari and L. McLaughlin, 'Optimal design of a condition-based maintenance model', in Reliability and Maintainability, 2004 Annual Symposium RAMS, 2004, pp. 528-533.

[28] D. Chen and K. S. Trivedi, 'Closed-form analytical results for condition-based maintenance', Reliab. Eng. Syst. Saf., vol. 76, no. 1, pp. 43-51, 2002.

[29] M. Finkelstein, Failure rate modelling for reliability and risk. Springer, 2008.

[30] J. G. Kemeny and J. L. Snell, Finite markov chains, vol. 28. Springer New York, 1976.

[31] F. Ball and G. F. Yeo, 'Lumpability and marginalisability for continuous-time Markov chains', J. Appl. Probab., pp. 518-528, 1993.

\section{Biographies}

Zhenglin Liang received the Ph.D. degree in reliability engineering and asset management from the University of Cambridge, U.K., in 2016. Currently, he is a research associate at the Distributed Information and Automation Laboratory at the University of Cambridge. His research interests include stochastic process, predictive maintenance, maintenance optimization and reliability of power equipment.

Ajith Kumar Parlikad received the Ph.D. degree in manufacturing management from Cambridge University, Cambridge, U.K., in 2006. He is now a Senior Lecturer in Industrial Systems with Cambridge University, Cambridge, U.K. His research interests include asset investment and maintenance decision making, particularly based on value. His research has been funded by the EPSRC and industry. He sits on the Executive Committee in the Institution of Engineering and Technology TPN on Asset Management, U.K., and is the Chair of the Academic and Research Network for the Institute of Asset Management, U.K.

Rengarajan Srinivasan is a research associate at the University of Cambridge's Department of Engineering. He obtained a $\mathrm{PhD}$ in engineering from the University of Cambridge and currently works on research activities in asset management at CSIC. His research interest is focused 
on value-based asset management and, in particular, optimising maintenance decisions based on value. He has worked with various industries to develop tools and techniques for asset management. Rengarajan has also published several papers in reputed academic journals and conferences and is a key member of the IAM Academic and Research Network.

Nipat Rasmekomen received the Ph.D. degree at the Institute for Manufacturing, University of Cambridge. He is based at the Institute for Manufacturing, where he is part of the Distributed Information and Automation Laboratory (DIAL). Nipat received his Bachelor degree in Engineering (1st class honours) from Chulalongkorn University, Thailand in 2008. He was then recruited into a post-graduate scholarship programme at PTT Public Company Limited, the national oil and gas company in Thailand. After that, he joined a Masters programme in Industrial Systems, Manufacturing and Management (ISMM, course 44) at the Department of Engineering, University of Cambridge, where he pursued the Ph.D. degree in Engineering, researching in the area of maintenance optimisation for complex engineering assets. 